\title{
Aspectos geodinámicos de un paleoestuario del desierto marginal de Chile. Río Copiapó ${ }^{1}$
}

\author{
María Victoria Soto Bäuerle², Joselyn Arriagada González², \\ Carmen Paz Castro Correa ${ }^{2}$, Michael Märker ${ }^{3}$ y Giuliano Rodolfi ${ }^{4}$
}

\begin{abstract}
RESUMEN
Las formas constituyentes del sistema estuarial del río Copiapó son analizadas durante el período de los últimos 30 años. Los trabajos de fotointerpretación y de terreno dan cuenta que durante tal período las formas esenciales del estuario no han sufrido cambios significativos, en tanto las del complejo estuarial, tales como la laguna estuarial, la playa arenosa y las dunas sí han presentado modificaciones. Los elementos morfológicos más significativos para la comprensión de estos fenómenos de cambio, han sido la hidrodinámica del oleaje y su correlación espacial con algunas componentes de la zona de surf y el continuum dunar. Las formas esenciales presentan una estabilización en su dinámica, lo que permite considerar al sistema estuarial del Copiapó, como un paleoestuario.
\end{abstract}

Palabras clave: Sistema estuarial, bancos estuariales, dunas costeras.

\begin{abstract}
In this investigation we analyze the evolution of forms that constitute the Copiapo River estuarine system during the past 30 years. Through photo interpretation and field work is possible to realize that during the period, the essential forms of the estuary haven't manifest significant changes, on the other hand, estuarine complex such as the estuarine lagoon, sandy beach and dunes have presented modifications. The most significant morphological elements in order to understand these phenomena of changes have been the dynamics of waves and their spatial correlation with the width of the surf zone and dune continuum. The basic shapes present stabilization in their dynamic, which allows us to consider the estuarine system of Copiapo, a paleoestuario.
\end{abstract}

Key words: Estuarial system, estuarine banks, coastal sand dunes.

El análisis de los sistemas estuariales de Chile desde el punto de vista geográfico y geomorfológico ha sido expresado a través de los trabajos clásicos de Pomar (1963), ArayaVergara (1970, 1981), Paskoff (1970); Paskoff

1 Proyecto FONDECYT No 1071098. Artículo recibido el 3 de julio de 2009 y aceptado el 12 de agosto de 2009.

2 Departamento de Geografía, Universidad de Chile (Chile). E-mail: mvsoto@uchilefau.cl; joarriag@uchile.cl; cpcastro@uchile.cl et al. (2003). En el marco del presente trabajo se plantea que los sistemas de desembocaduras en las costas de Chile central y del semiárido deben ser abordados a través de esquemas evolutivos y taxonómicos adecuados a la

3 Institut für Geoökologie, Universität Potsdam (Alemania). E-mail: michael.maerker@geographie.unituebingen.de

4 Dipartimento Scienza del Suolo e Nutrizione della Pianta, Università degli studi di Firenze (Italia). Email: giuliano.rodolfi@unifi.it 
realidad morfoclimática actual y a las formas heredadas del Cuaternario. En este contexto Araya-Vergara (1981) concluye respecto de la marcada influencia morfoclimática en los ambientes de desembocadura, reconociendo la siguiente zonificación:

a) Zona de los deltas distales: corresponde a la influencia morfoclimática del desierto, con aportes estivales derivados de influencias halógenas de altura.

b) Zona de las rías progradadas: aquellas que coinciden con las condiciones semiáridas, desde el río Copiapó $\left(27^{\circ} 20^{\prime}\right.$ latitud Sur) hasta el Maipo inclusive $\left(33^{\circ} 47^{\prime}\right.$ latitud Sur). Estas paleorrías determinadas por Paskoff (1970) muestran un relleno más perfecto, poco ponderado por el sistema fluvial actual. Ello refleja a su vez la carga sólida superior que se atribuye a los ríos de zonas semiáridas.

c) Zona de los deltas estuariales: corresponde al área de transición entre el semiárido y el subhúmedo, desde la desembocadura del río Rapel ( $34^{\circ}$ latitud Sur) y la del Biobío ( $37^{\circ}$ latitud Sur) inclusive, donde la energía fluvial es importante para calibrar canales amplios de curso inferior y para aportar abundante carga de masa, pero la energía marina también es suficiente como para impedir el progreso de la progradación hacia el mar.

d) Zona de las rías: corresponde a aquellas vinculadas con las condiciones húmedas de la Región de Los Lagos.

El modelo teórico y conceptual de ArayaVergara (1981) ha sido aplicado a dos sistemas estuariales. En el caso del Aconcagua se concluyó que corresponde a un estuario dominado por procesos fluviales y del oleaje (Cortez, 2002; Martínez \& Cortez, 2007), mientras que el río Maipo da cuenta de un sistema caracterizado por una tendencia a ser dominado por la acción fluvial, pero evidenciando una alternancia de efectos dominantes dependiendo de la estacionalidad (Arriagada, 2005).

La determinación de tal clasificación zonal de los sistemas estuariales fue establecida según la presencia y estado de las formas esenciales de cada subsistema: zona fluvial o proximal, con meandros estuariales; zona deltaica interior o media, con bancos medios y distributarios; zona lagunar o distal, con laguna distal semibloqueada por barrera litoral o flecha, una barrera o flecha exterior (ArayaVergara, 1981). De acuerdo a lo anterior, cobra relevancia la identificación y evolución temporal y espacial de las formas esenciales señaladas, cuya presencia, formas y estados permiten establecer el factor dominante en su morfología.

De acuerdo a tal diversidad de formas y a la complejidad de una definición taxonómica única (hidrológica, geomorfológica, oceanográfica y/o ecológica), surge también el planteamiento de considerarlas como un sistema integrado, pudiendo ser asimilados a las barreras (Dillenburg, 2009). En esta línea de pensamiento Dillenburg \& Hesp (2009) definen el sistema de barrera como una línea litoral con playa, cordones de dunas anteriores y campo de dunas asociado. Siendo así, el sistema de desembocadura del río Copiapó constituye genéricamente una barrera, en la medida que los elementos morfológicos encontrados están asociados al aporte de sedimentos, a la condición del viento, de las olas y mareas y al emplazamiento geológico y formas fluviales; estas son formas descritas para el Copiapó por Paskoff y Manríquez (2004). No obstante, el mismo autor (Paskoff, 1998) define un estuario según sus componentes morfológicas (fluvial y marina) de manera coincidente con las formas descritas para el sistema estudiado.

En relación a cómo definir un sistema de desembocadura, ya sea como estuario, delta o delta estuarial, la discusión no está concluida, toda vez que Prandle et al. (2006) plantea la necesidad de elaborar nuevas tipologías para las formas estuarinas, modelando para ello la forma en que la batimetría se ajusta a un cambio del nivel del mar, a la amplitud de marea, al flujo fluvial y al abastecimiento sedimentario, asuntos importantes de analizar en función de los niveles diferenciales de intervención antrópica en las cuencas hidrográficas y el retroceso y degradación de los sistemas ecológicos fluvio marinos (Lester \& Fairweather, 2009). El aporte realizado por los autores citados para un estuario de ambiente semiárido, da cuenta de la degradación del mismo debido a la extracción 
de agua, contaminación y la eutroficación y concluye con la necesidad de acciones de manejo para redireccionar los procesos actuales. El impacto de la construcción, urbanización e instalación de obras en el estuario del río Sena, Francia, denota una velocidad de respuesta muy alta, en términos de la erosión y depositación diferencial en relación al tipo y localización específica de la intervención realizada (Cuvilliez et al., 2009).

El contexto geográfico en que se inserta la problemática específica del estuario del Copiapó dice relación con una cuenca hidrográfica sujeta a profundos y acelerados cambios de uso del suelo e intensidad del mismo, que ha generado un aumento de la vulnerabilidad física del territorio asociado a las transformaciones en las formas del paisaje, presentando una mayor disponibilidad potencial de masa en el sistema, posible de ser transferida du- rante los eventos El Niño (Castro et al., 2009).

Consecuentemente, el impacto de los eventos naturales normales y excepcionales son elementos a considerar, tal como lo demuestra el análisis de Federici y Rodolfi (2001, 2004), quienes establecieron que el rápido retroceso de la línea de costa en Ensenada de Atacames (Ecuador) se debe a la erosión provocada por los eventos El Niño, pero también a la acción indirecta de la destrucción de los humedales.

De acuerdo a los antecedentes anteriores, el propósito de esta investigación es identificar y caracterizar las formas esenciales y los factores que operan en un sistema estuarial localizado en un ambiente morfoclimático de desierto, estableciendo las condiciones de funcionamiento dinámico en función a la presencia de formas heredadas y actuales.

Figura $\mathrm{N}^{\mathrm{o}} 1$

Área de estudio

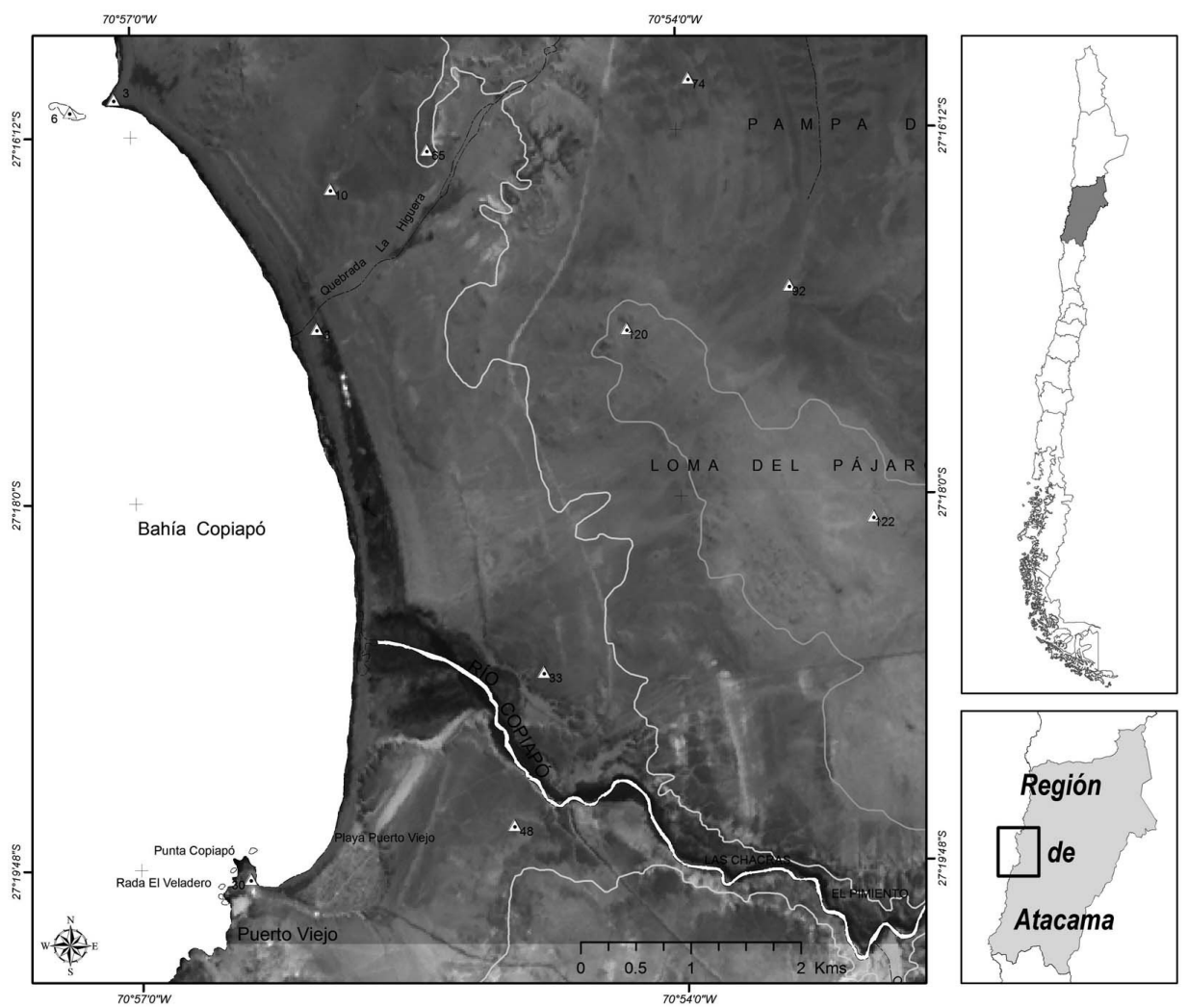

Fuente: Elaboración propia en base a información IGM. 


\section{Antecedentes del área de estudio}

El estuario del Copiapó (Figura $N^{0} 1$ ), en la Región de Atacama ( $27^{\circ} 12^{\prime}$ latitud Sur- $70^{\circ} 55^{\prime}$ longitud Oeste), se presenta esculpido en una amplia unidad de planicies litorales correspondientes a terrazas pleistocénicas, compuestas por secuencias marino-litorales y fluvio-estuarinas (Godoy et al., 2003). Estas formas han sido modeladas como una sucesión de terrazas escalonadas, de unos 20 kilómetros de ancho y con, al menos, seis relictos de terrazas costeras, las que además han sufrido un alzamiento tectónico durante el Cuaternario (Quezada et al., 2007).

En las extensas planicies costeras elevadas existen prominentes cordones litorales inactivos, con una marcada curvatura, correspondientes a playas en bahía, que evidencia una antigua posición de la línea de costa y la evolución de la misma. Estas formas dan cuenta de los procesos de progradación/ erosión glacioeustática y los efectos de la tectónica en esta parte del territorio nacional. A este respecto, Araya-Vergara (2008) ha concluido que los niveles de solevantamiento de la costa pacífica son mayores que el Atlántico y que las superficies elevadas de terrazas son formas derivadas de la fragmentación tectónica.

La conformación de las terrazas de Atacama es la resultante de variaciones eustáticas del mar y alzamiento tectónico, destacando en este contexto geocronológico el desarrollo de una amplia terraza cuya edad corresponde a 0,86 $\pm 0,11 \mathrm{Ma}$. Dentro de estos rangos se identificaron tres interestadiales (MIS 19 (780 ka), MIS 21 (860 ka) y MIS 25 (950 ka)), donde se destaca el interestadial MIS 11 (400 ka), cuando se produce erosión en las terrazas costeras, incluyendo las más antiguas (Quezada et al, 2007). Marquardt et al. (2004) asignaron los estadios isotópico MIS 11, MIS 9 (330ka), MIS 7 (210 ka) y MIS 5 (125 ka) a las terrazas marinas localizadas en cotas inferiores a los 200 m.s.n.m.

\section{Metodología}

El abordaje metodológico consistió en la descripción y análisis de las formas del sistema estuarial a través de fotointerpretación de fotografías aéreas, para un período de 30 años. Se realizó trabajo en terreno. Esta información se sistematizó a través de la confección de la carta geomorfológica del sistema de desembocadura.

El análisis de la morfodinámica temporoespacial se realizó a partir de la identificación de las formas esenciales constituyentes de un sistema estuarial, tales como los meandros y bancos estuariales, bancos laterales cuspidados, laguna estuarial y flecha litoral, de acuerdo a la sistemática de Araya-Vergara (1981).

Las relaciones genético-evolutivas existentes entre el litoral cercano y las playas arenosas que evidencian la importancia de los procesos derivados del mar de fondo, pueden ser deducidas a partir de la clasificación de las olas. Concordantemente, este aspecto fue analizado según el tipo de playa dominado por olas, según la sistematización de Wrigth \& Short (Short, 1999), las que se clasifican en playas disipativas, intermedias y reflectivas.

Playas disipativas: playas cuya zona de surf es rasa, con una baja gradiente y presentan al menos tres líneas de rompientes.

Playas intermedias: son las playas más comunes y corresponden a tipo intermedio entre disipativas de alta energía y reflectivas de baja energía; se subdivide en cuatro tipos: barra y surco longitudinal (LBT); playa y barra rítmica (RBB); barra y rips transversal (TBR); y terraza de bajamar (LTT).

Playas reflectivas: playas con una fuerte gradiente, con una zona de saca y resaca (swash zone) relativamente estrecha.

En el contexto de las relaciones existentes entre el litoral cercano y la condición dinámica de la paya arenosa de la desembocadura, se utilizó la clasificación anterior y la identificación de las corrientes de playa, como factor explicativo de la transferencia de masa en la playa (Massenlik et al., 2006). 
En esta misma línea de análisis, la consideración de las dunas fue especialmente relevante en la medida que constituyeron las formas indicativas de transferencia de masa. Para poder establecer la condición de cambio, se analizaron los tipos de formas y su inclusión en el continuum dunar (Figura $N^{\circ}$ 2) de Araya-Vergara. Se comparó el estado de las dunas en fotos aéreas desde el año 1979 hasta su estado actual, en terreno, estableciendo las condiciones de transmutación y transmudación de las mismas (Araya-Vergara, 1987).

Figura $\mathrm{N}^{\circ} 2$

Continuum dunar y familias de dunas

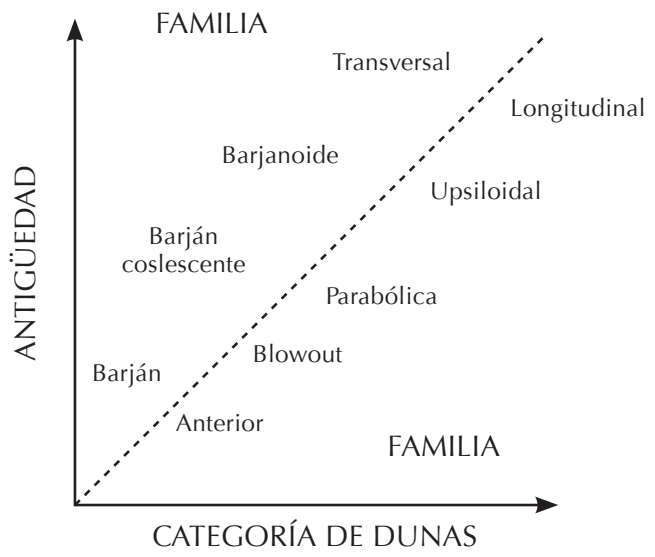

Fuente: Araya-Vergara, 1987.

Se midió el ancho de la zona de rompiente según método de Araya-Vergara (1996), en la parte proximal, media y distal de la ensenada, en un período de 30 años. Cabe destacar que si bien estas mediciones se realizaron con fotos aéreas para un día, mes y año determinado, han constituido una herramienta indicativa de tendencias en el tipo de zona de rompiente y su relación con la dinámica existente en la zona de surf.

Los patrones de cambio y tendencias evolutivas se analizaron a través de la comparación temporal de cartas topográficas y fotografías aéreas (1979 a 2008), distinguiendo la zona proximal, media y distal de este sistema de desembocadura.

\section{Resultados}

\section{Marco geomorfológico}

El sistema estuarial del río Copiapó se inscribe en un marco paleogeográfico de marcada connotación cuaternaria, cuyo paisaje característico de terrazas marinas, de desierto marginal, contrasta con un fondo de valle incidido de clara morfología fluvial. Las relaciones genéticas del modelado marino y fluvial son el rasgo distintivo del paisaje heredado del ambiente marino reinante en la zona (Figura $N^{\circ} 3$ ).

El curso fluvial del río Copiapó en la zona de las planicies costeras se encuentra circunscrito por al menos tres niveles de terrazas marinas claramente identificables, al igual que aquellas formas polifásicas de origen fluvial (Figura $N^{\circ} 4$ ), esculpidas en las planicies litorales cuaternarias como garganta epigénica. Las superficies de terrazas presentan una importante cobertura eólica que ha sido estudiada por Araya-Vergara (2001), quien señala que el erg de Atacama ha sido conformado por arenas aportadas desde costas pleistocénicas, pero con evolución holocénica, cuya masa arenosa mantiene relaciones geosinergéticas con una cuenca fluvial con escurrimiento perenne hacia el mar, en un ambiente de desierto árido, con actividad dinámica. A su vez, Paskoff et al. (2003) señalan que si bien las dunas de Atacama son formas vivas, constituyen dunas remanentes, pues la fuente de abastecimiento estaría agotada, siendo esta de origen fluvial.

En lo que concierne al estuario del Copiapó, se puede distinguir claramente la terraza baja, la cual representa el lecho máximo de inundación (máximo de la transgresión flandesa). El complejo estuarial está conformado por una extensa laguna estuarial que se desplaza en dirección norte, separando la playa arenosa actual y remanentes de laguna localizados junto al borde del escarpe de la terraza marina principal.

En la actualidad, la laguna estuarial contiene una no despreciable lámina de agua, con abundante vegetación halófita, con intrusiones de vegetación de agua dulce. La presencia de bancos y costras salinas evidencian el retroceso de la misma y las condiciones de 
vulnerabilidad ambiental a que está sometida la laguna (humedal de Copiapó).

\section{Formas esenciales del estuario del Copiapó}

Las formas esenciales presentes en el estuario del Copiapó, identificadas a través de fotointerpretación evolutiva y reconocimiento en terreno, son los bancos laterales cuspidados asociados a los meandros estuariales, los bancos medios estuariales y una laguna estuarial. Los meandros estuariales deben sus características a la acción de las corrientes de flujo y de reflujo, las que están relacionadas con la acción fluvial y la acción marina. Presentan como característica distintiva la forma de estos bancos, ancha en la parte central y angosta en los extremos. Estos bancos no han presentado cambios en su morfología en el período analizado.

Las bancos medios estuariales (Figura $\mathrm{N}^{\circ}$ 5) también pueden ser claramente identificados y clasificados como tales, en la medida que la forma cuspidada heredada característica fue determinada por la influencia tanto del oleaje como por la acción fluvial. Al igual que el caso anterior, no se hallaron evidencias de cambio en las formas y en su posición dentro del sistema estuarial durante el período de tiempo en estudio. Esta afirmación se condice con la condición actual de estos bancos, los cuales se encuentran absolutamente estabilizados.

Figura $\mathrm{N}^{\circ} 3$

Geomorfología del sistema de desembocadura y playa del río Copiapó

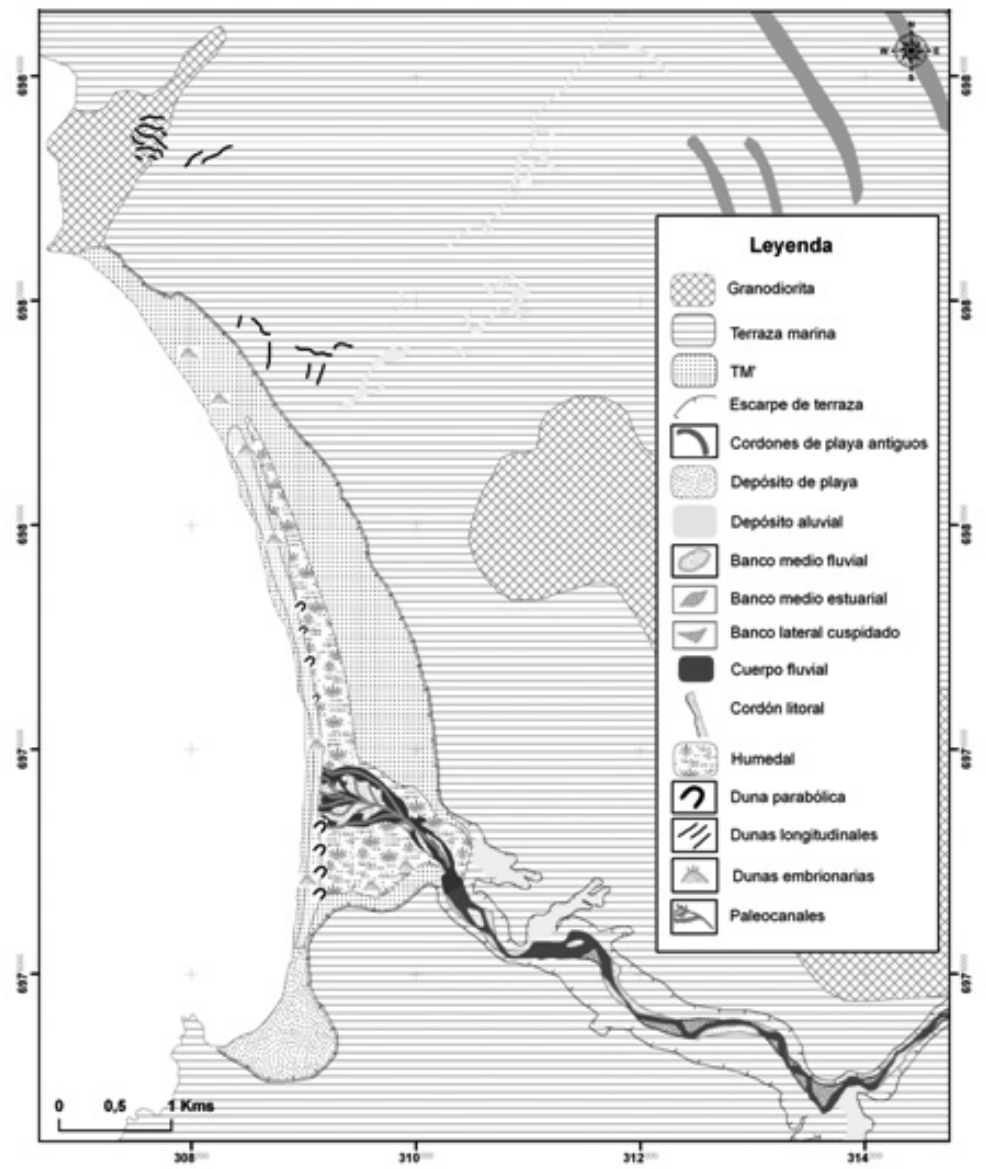

Fuente: Elaboración propia. 
Figura $\mathrm{N}^{\circ} 4$

Terrazas fluvio-marinas y talweg del río Copiapó*

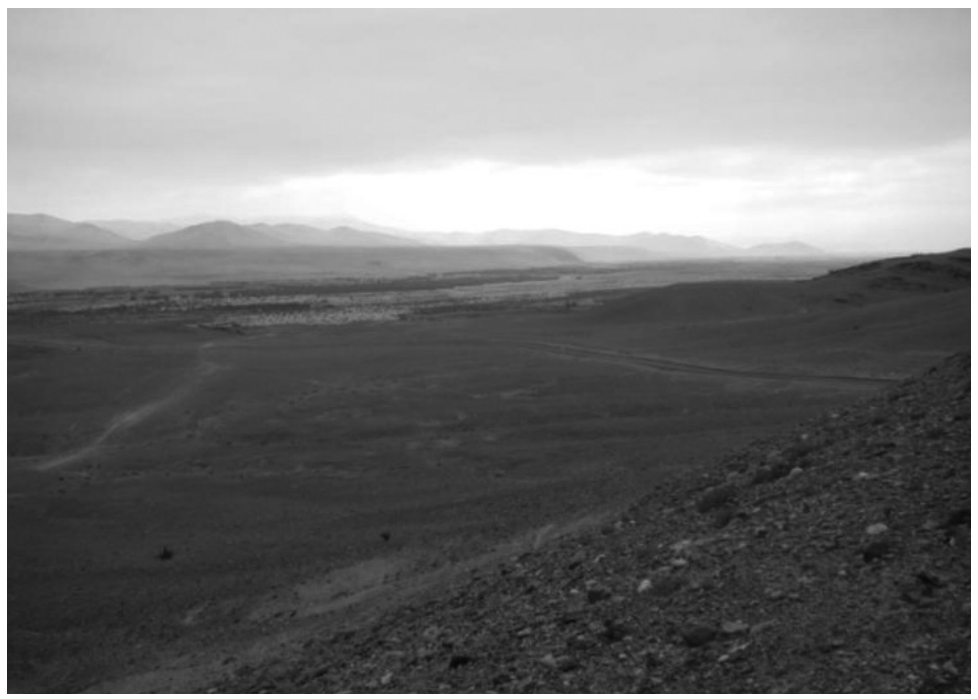

*Fotografía tomada desde un tercer nivel de terraza, en que se observan rodados alterados. Diciembre de 2007. Fuente: Colección personal de los autores.

Figura $\mathrm{N}^{\circ} 5$

Meandros estuariales y bancos laterales cuspidados

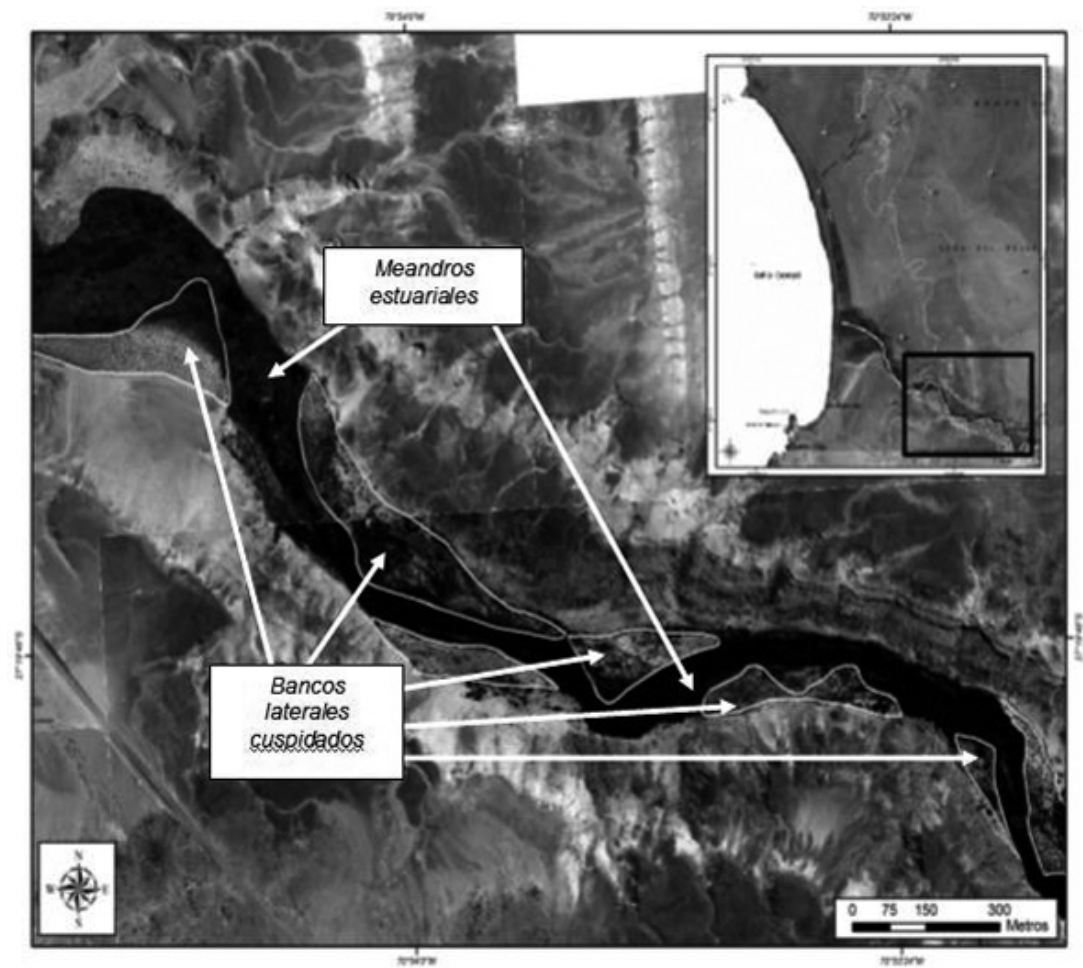

Fuente: Elaboración propia. 
La laguna estuarial se presenta bloqueada producto del cierre de la flecha litoral (Figura $\mathrm{N}^{\circ}$ 6). Esta flecha litoral corresponde a un alargamiento del cordón litoral del sur del estuario, el cual se abastece de sedimentos transportados por la deriva litoral. En el período estudiado, no se encontraron vestigios de que el estuario se haya presentado abierto; tal situación refleja la influencia del transporte longitudinal de carga actuando en una playa con sedimentos suficientes en las inmediaciones de la desembocadura del río, el que a su vez presenta un caudal insuficiente para mantener abierta la desembocadura.

\section{Características del litoral cercano del estuario del Copiapó}

Se evidenció el marcado predominio temporal y espacial de características relacionadas a playas intermedias, sobre todo del tipo barra y rip transversal (TBR) y barra y surco longitudinal (LBT). Esta característica de la geodinámica del litoral cercano en el sistema estudiado, da cuenta de la presencia de patrones de rompientes y corrientes de playa asociados, tanto perpendiculares como paralelos a la línea de costa (Figura $\mathrm{N}^{\circ}$ 7). Las condiciones del oleaje de carácter transversal y longitudinal coexisten en los diferentes tipos de playas, dando cuenta así que la presencia sistemática de corrientes de playa constituyen un hecho relevante para la morfosedimentología del sector, asociada a la transferencia de sedimentos a través de la corriente de deriva de playa (longshore current) y las corrientes transversales de rip.

La condición intermedia de carácter longitudinal asociada a las partes proximales se asocia entonces con la trasferencia de sedimentos desde la desembocadura hacia el norte, y los patrones transversales de la zona media y distal indican la presencia de características apropiadas para la transferencia de sedimentos hacia la zona intermareal y la anteplaya de esa parte de la ensenada. La presencia de dunas anteriores actuales y la condición de avance, que se demuestra con la presencia de dunas parabólicas hacia el humedal, son las formas indicativas del abastecimiento actual de masa.

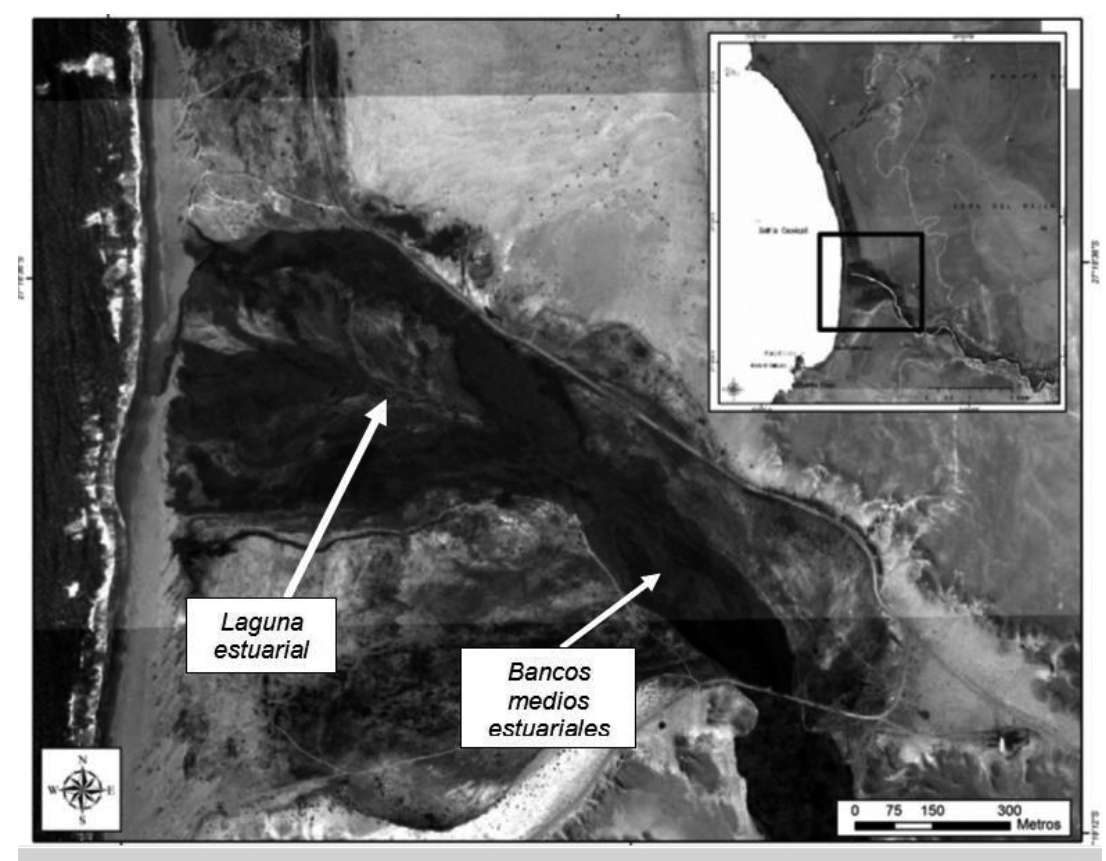

Fuente: Elaboración propia. 
Figura $\mathrm{N}^{\circ} 7$

Tipos de playas dominadas por olas, bahía de Copiapó
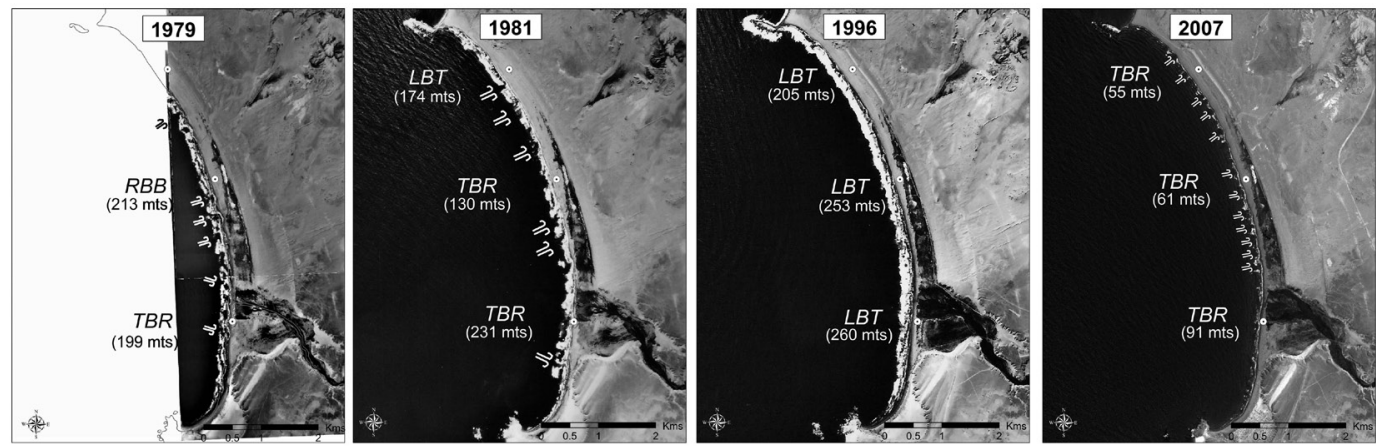

$T B R=$ Barra y Rips Transversal; $R B B=$ Barra y Playa Rítmica; $L B T=$ Barra y Surco Longitudinal. Fuente: Elaboración propia.

Sin embargo, al analizar el ancho de las zonas de rompientes (Cuadro $\mathrm{N}^{\circ} 1$ ) se aprecia una tendencia a la mayor amplitud en la zona media y proximal, sobre todo a partir del año 1981. Esta situación fue además constatada en la campaña de terreno de invierno y verano de 2007 y 2009. A la vista de esta información es posible establecer que para los dos últimos períodos existe una tendencia asociada a una mayor condición de sedimentación en la zona proximal y media, la que disminuye hacia la zona distal.

La observación de la zona del litoral cercano, específicamente de la zona de las rompientes, vista a escala de detalle en terreno, evidencia la presencia de mega cusps en la parte media de la ensenada, en las zonas de mayor espesor de dunas en la playa actual, coincidentemente con trenes de olas curvados hacia la playa e interrumpidos en la parte central del cusps, patrón característico de costas transversales, de alta energía y presencia de mega rips.

Cuadro $N^{\circ} 1$

Ancho de zona de rompiente, diferentes años

\begin{tabular}{|l|c|c|c|c|}
\hline Zona/Año & $\begin{array}{c}1979 \\
(\mathrm{~m})\end{array}$ & $\begin{array}{c}1981 \\
(\mathrm{~m})\end{array}$ & $\begin{array}{c}1996 \\
(\mathrm{~m})\end{array}$ & $\begin{array}{c}2007 \\
(\mathrm{~m})\end{array}$ \\
\hline Distal & - & 174 & 205 & 55 \\
Media & 213 & 130 & 253 & 61 \\
Proximal & 199 & 213 & 260 & 94 \\
\hline
\end{tabular}

Fuente: Elaboración propia.

\section{Dunas costeras}

Las dunas asociadas a la desembocadura del río Copiapó presentan una evolución particularmente interesante, en la medida que dan cuenta de transformaciones ocurridas en los últimos 30 años. La observación de las dunas en la imagen de 1979 y 1980, expresa una ensenada en cuyas partes distal y media se encontraban los mayores espesores de dunas activas, presentando patrones vinculados con el continuum dunar de las antedunas.

En la parte distal, no obstante lo anterior, existía un cuerpo menor de dunas transversales, y a barlovento de estas, dunas longitudinales activas, con unos patrones de cordones muy bien definidos e individualizados. En ese mismo período, en la zona media, la laguna del río Copiapó se presentaba bastante amplia y bien desarrollada hacia el norte de la ensenada; la anteplaya asociada a esta sección era muy estrecha. De acuerdo a las formas y patrones ya descritos, se puede señalar que existían evidencias morfológicas de un abastecimiento de arenas en las tres secciones de la ensenada, de la misma manera que de aprovisionamiento permanente de agua al humedal desde el río Copiapó.

La situación actual es bastante diferente y expresa una condición de escaso y diferencial abastecimiento espacial de arenas a lo largo de la costa. La parte distal de la ensenada presenta un extenso sector de dunas 
anteriores alargadas, y las dunas secundarias transversales se presentan bien conservadas. Hacia el interior, a barlovento, por sobre la terraza marina, probablemente correspondiente al nivel MIS 5 (125 ka), los cordones de dunas longitudinales han desaparecido, siendo reemplazados por amplios cordones de dunas alargadas vegetadas y montículos deflacionarios actuales. Las dunas parabólicas descritas para el año 1979 y 1981, han sido profundamente deflacionadas evolucionando a dunas upsiliodales.

Actualmente se aprecia una intensa acción de las dunas anteriores en la anteplaya de toda la ensenada, pero siendo más expresiva en

Figura $\mathrm{N}^{\circ} 8$

Microdunas, dunas anteriores y dunas parabólicas. Sector proximal de la ensenada

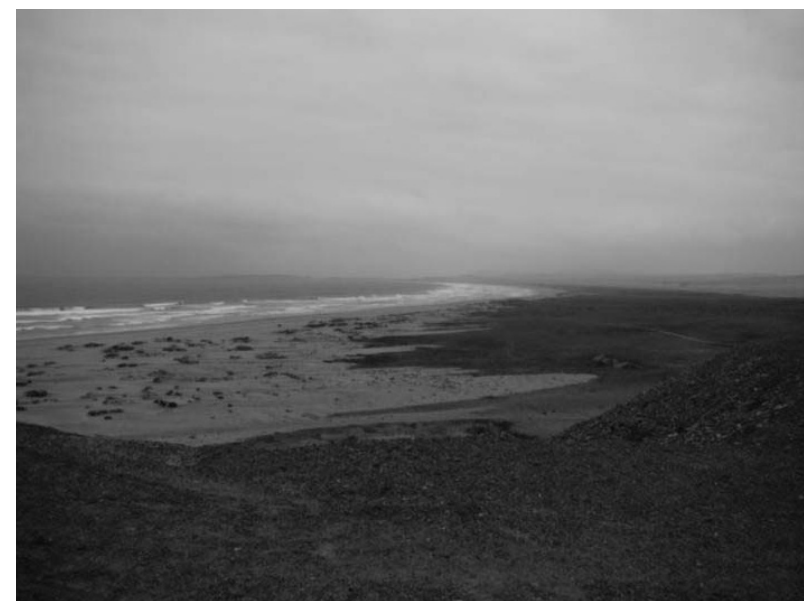

Fuente: Colección personal de los autores.

Figura $N^{\circ} 9$

Cabalgamiento de dunas en escarpe de terraza, con ripple marks.

Sector proximal de la ensenada

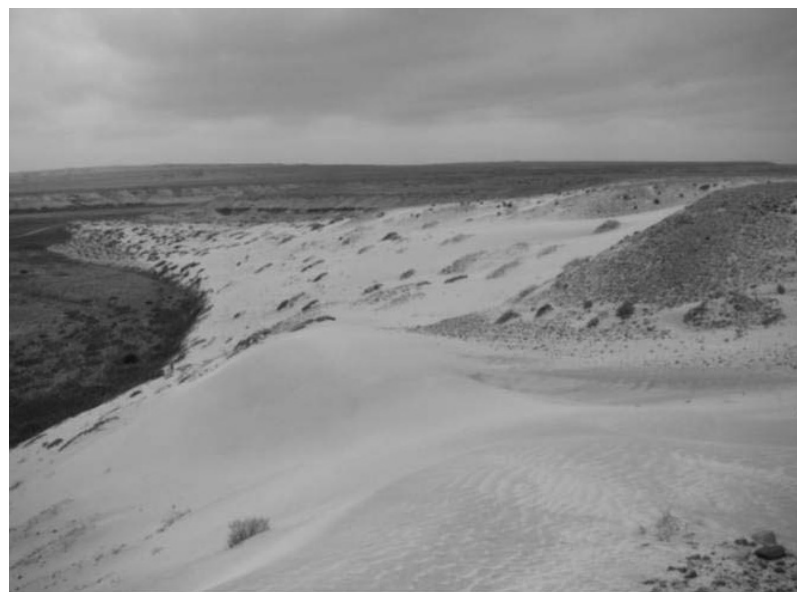

Fuente: Colección personal de los autores. 
la parte proximal y media, avanzando hacia el humedal, presentando ahí un claro patrón parabólico (Figura $N^{\circ} 8$ y Figura $N^{\circ} 9$ ), que indica que existe abastecimiento de arenas.

\section{Consideraciones finales}

El marco geomorfológico de la zona estuarial del río Copiapó da cuenta de una complejidad de sistemas dinámicos costeros actuales y heredados, constituyendo de tal forma un paisaje de síntesis de los procesos de la cuenca a lo largo de su historia geológica y geográfica.

De acuerdo al análisis evolutivo de las formas que componen el sistema se puede señalar que en los últimos 30 años ha habido patrones diferenciales de cambio. En este contexto las formas que presentan condiciones de estabilización son los bancos estuariales, toda vez que se aprecia la existencia de suelo y vegetación.

No obstante lo anterior, de acuerdo a los antecedentes aportados por los estudios de Marquardt et al. (2004) y Quezada et al. (2007), en las terrazas marinas del entorno directo del sistema de desembocadura se asume la presencia de un paisaje constituido por formas antiguas heredadas, las que han sido datadas por métodos absolutos como de edad cuaternaria. Asimismo, la incisión de tipo meandrante en la terraza baja está asociada a los movimientos corticales aludidos por el mismo autor.

Las formas que sí han presentado un cambio están asociadas directamente con la dinámica que existe en la desembocadura del río Copiapó; esta se encuentra cerrada por una extensa barrera, con un pequeño canal exutorio. Tal situación podría explicarse por el mayor aporte de sedimentos desde la cuenca, sumado al bajo caudal del río Copiapó.

Sin embargo, el sistema de desembocadura no solo está conformado por las formas de bancos estuariales de origen fluvial, sino que por la laguna estuarial, las dunas y las relaciones con la hidrodinámica de la playa. Al analizar estas formas, se aprecia que ha habido cambios, y que si bien estos no son significativos desde el punto de vista areal, lo son desde una perspectiva evolutiva.
El análisis conjunto de las relaciones existentes entre la zona de surf y el tipo de playa, según taxonomía de Short (1999), proporcionó los antecedentes que permiten comprender la dinámica actual de transferencia de masa en la línea de costa asociada a la desembocadura del río Copiapó. En este contexto, la concordancia existente entre la mayor amplitud de la zona de rompiente y el ancho del sistema de dunas concuerda con lo observado en otras líneas de costa por ArayaVergara (1996), Soto $(2003,2005)$, Soto y Arriagada (2007), en términos de aporte sedimentario efectivo desde la zona de surf a la playa.

No obstante ello, es muy especial la localización de estas formas correlacionadas morfosedimentológicamente, pues los autores antes citados, en ensenadas de Chile central establecieron que tales relaciones se localizaban preferentemente en las partes distales o medias, pero no en la zona proximal de una bahía. Si se considera también los tipo de playas intermedias de Barra y Surco Longitudinal (LBT) y de Barra y Rip Transversal (TBR), que favorece el transporte la primera y la depositación/erosión la segunda, ello corrobora que la transferencia de masa se realiza preferentemente y en mayor abundancia en la zona proximal.

Este hecho se comprueba al verificar las condiciones de evolución dunar, que ha sido de mayor importancia relativa en dichas zonas, en detrimento de la parte distal, en que si bien la estructura de barras y surcos en disposición transversal y los rip, demuestran la condición de depositación, esta es menos expresiva.

Consecuentemente, la zonal distal no ha estado recibiendo abastecimiento de arenas desde la zona de surf, lo que además queda reflejado por las evidencias de procesos de transmutación y trasmudación de las dunas, que están evolucionando a amplios campos de deflación. En este contexto, es importante destacar los evidentes procesos de transmudación, principalmente en dunas longitudinales, y transmutación en las dunas parabólicas-upsiloidales.

Cabe destacar que en la actualidad la laguna estuarial no existe como tal, sino que se 
encuentra con un aporte de agua muy bajo. Otra consideración importante, es la continua alimentación que tienen las terrazas, de material eólico, el cual las ha ido recubriendo de forma constante, asociado tal vez al mayor aporte de sedimentos al litoral.

Consecuentemente, los resultados arrojan la presencia de un paleoestuario bloqueado por la acción energética del oleaje, cuyas formas esenciales fueron originadas en situaciones climáticas diferenciales a las actuales. La aplicación del término paleo se aplica en el contexto de formas desarrolladas en un tiempo pasado y en un medio ambiente climático diferente del actual; otra acepción está referida a los cambios que la desembocadura haya desarrollado, ya sea por causa de variaciones climáticas, movimientos estáticos, por neotectónica, crecidas anómalas y efectos antrópicos. Todos estos aspectos son reconocidos en la desembocadura del río Copiapó, según los antecedentes de las dataciones y los cambios observados en los últimos 30 años. Los fenómenos de transmudación y transmutación actuales en la parte distal se condicen con lo anterior, por cuanto el aporte de sedimentos solo se produce en las partes proximales.

En base a los antecedentes antes expuestos y en el contexto de un sistema geográfico integral como la cuenca del río Copiapó, la tendencia evolutiva esperable es a la progradación de la barrera y retroceso de la laguna estuarial, el desarrollo de antedunas y su evolución a dunas parabólicas, avanzando hacia la laguna y por sobre las terrazas en la parte proximal. En la parte media y distal, una intensa deflación y avance de las dunas hacia las terrazas, asociados a intensos procesos evolutivos de transmudación y transmutación, en función de los aportes de masa desde la cuenca y la trasferencia en la zona litoral.

Finalmente, es necesario enfatizar el contexto geográfico en el que se inserta el estuario del Copiapó, el cual dice relación con una cuenca hidrográfica sujeta a profundos y acelerados cambios de uso del suelo e intensidad del mismo, que está generando un aumento de la vulnerabilidad física del territorio asociado a las transformaciones en las formas del paisaje. En este sentido, los fenómenos acrecionales naturales evidenciados para el área de estudio también podrían estar asociados a las actividades económicas de la cuenca, minería y agricultura de exportación.

\section{Referencias bibliográficas}

ARAYA-VERGARA, J. F. Contribución al estudio de los procesos estuariales en las desembocaduras de los ríos Rapel y Maipo. Revista Informaciones Geográficas, 1970, № 20, p. 17-38.

ARAYA-VERGARA, J. F. El concepto de delta en ría y su significado en la evolución litoral (ejemplo de Chile central). Revista Informaciones Geográficas, 1981, № 28, p. 19-42.

ARAYA-VERGARA, J. F. The evolution of modern coastal dune systems in central Chile. In: GARDINER, V. (ed.). International Geomorphology 1986. Proceedings of the First International Conference on Geomorphology Part II. Chichester: John Wiley \& Sons, 1987, p. 1231-1244.

ARAYA-VERGARA, J. F. Sistema de interacción oleaje-playa frente a los ergs de Chanco y Arauco, Chile. Gayana Oceanología, 1996, vol. 4, № 2, p. 159-167.

ARAYA-VERGARA, J. F. Los ergs del desierto marginal de Atacama. Revista Informaciones Geográficas, 2001, №35, p. 27-66.

ARAYA-VERGARA, J. F. Toward a morphotectonic model of the South-American coasts. Revista Brasileira de Geografía, 2008, (en prensa).

ARRIAGADA, J. Cambios en el sistema estuarial del Maipo y su relación con obras portuarias, Chile Central. Memoria título de Geógrafo. Santiago: Escuela de Geografía, Facultad de Arquitectura y Urbanismo, Universidad de Chile, Chile, 2005.

CASTRO, C. P.; SOTO, M. V.; FERNÁNDEZ, R.; MÄRKER, M. y RODOLFI, G. Impacto en la geodinámica actual del valle de Nantoco, cuenca del río Copiapó, asociado a la reconversión productiva. Revista de Geografía Norte Grande, 2009, № 42, p. 81-99. 
COOPER, J. A. Geomorphological variability among microtidal estuaries from the wave-dominated South African coast. Geomorphology, 2001, N 40, p. 99-122.

COOPER, J. A. Geomorphology of Irish estuaries: inherited and dynamic controls. Journal of Coastal Research, 2006, № 39, p. 176-180.

CORTEZ, C. Observaciones dinámicas y geomorfológicas en el estuario del Aconcagua, Chile Central. Memoria título de geógrafo. Santiago: Escuela de Geografía, Facultad de Arquitectura y Urbanismo, Universidad de Chile, Chile, 2002.

CUVILLIEZ, A.; DELOFFRE, J.; LEFITE, R. \& BESSINETON, C. Morphological responses of an estuarine intertidal to constructions since 1978 to 2005: TLE Seine estuary (France). Geomorfology, 2009, N 104, p. 165-174.

DALRYMPLE, R.; ZAIMLN, B. \& BOYD, R. Estuarine facies models. Conceptual basis and stratigraphic implications. Journal of Sedimentary Petrology, 1992, N 62, p. 1130-1146.

DILLENBURG, S. Workshop Geologia Marinha-40 anos do CECO. En: Porto Alegre, Universidade Federal do Rio Grande do Sul, Instituto de Geociências, Centro de Estudos de Geologia Costeira e Oceânica, 2009.

DILLENBURG, S. \& HESP, P. Geology and Geomorphology of Holocene coastal barriers of Brazil. Heildelberg: Springer-Verlag, 2009.

FEDERICI, P. \& RODOLFI, G. Rapid shorelines retreat along the Esmeraldas Coast, Ecuador: natural and man-induced processes. Journal of Coastal Conservations, 2001, N ${ }^{\circ} 7$, p. 163-170.

FEDERICI, P. \& RODOLF, G. Geomorphological features and evolution of the Ensenada de Atacames (Provincia de Esmeraldas, Ecuador). Journal of Coastal Research, 2004, vol. 20, $\mathrm{N}^{\circ} 3$, p. 700-708.

GODOY, E.; MARQUARDT, C. y BLANCO, C. Carta Geológica de Chile. Carta Caldera. Región de Atacama. Santiago: Servicio Nacional de Geología y Minería, Serie Geología Básica N 76, 2003, p. 1-38.
LESTER, R. \& FAIRWEATHER, P.G. ModeIling future conditions in the degraded semiarid estuary of Australia's largeste river using ecosystem states. Estuarine, Coastal and Shelf Science, 2009, N85, p. 1-11.

MARQUARDT, C.; LAVENU, A.; PORTLIEB, L.; GODOY, E. \& COMTE, D. Coastal neotecthonics in southern central Andes: uplift and deformation of marine terraces in norther Chile $\left(27^{\circ} \mathrm{S}\right)$. Tectonophysics, 2004, No394, p. 193-219.

MARTÍNEZ, C. y CORTEZ, C. Características hidrográficas y sedimentológicas en el estuario del río Aconcagua, Chile central. Revista de Geografía Norte Grande, 2007, Nº 37 , p. 63-74.

MASSELINK, G.; KROON, A. \& DAVIDSON-ARNOTT, R. Morphodynamics of intertidal bars in wave-dominated coastal settings-a review. Geomorphology, 2006, N ${ }^{\circ}$ 73, p. 33-49

PASKOFF, R. Recherches geomorphologiques dans le Chili semi-aride. Bordeaux: Biscaye Frères, 1970.

PASKOFF, R. Les Litoraux. Impact des aménagesment sur leur evolution. París: Armand Colin, 1998.

PASKOFF, R.; CUITIÑO, L. y MANRÍQUEZ, H. Origen de las arenas dunares de la región de Copiapó, desierto de Atacama, Chile. Revista Geológica de Chile, 2003, vol. $30, N^{\circ} 2$, p. 355-361.

PASKOFF, R. y MANRÍQUEZ, H. Las dunas de las costas de Chile. Santiago: Instituto Geográfico Militar, 2004.

POMAR, J. Cambios en los ríos y en la morfología de la costa de Chile. Revista Chilena de Historia y Geografía, 1963, No 130, p. 318-356.

PRANDLE, D.; LENE, A. \& MANNING, A. New tipologies for estuarine morphology. Geomorphology, 2006, № 81, p. 309-315.

QUEZADA, J.; GONZÁLEZ, G.; DUNAI, T.; JENSEN, A. y JUEZ-LARRÉ, J. Alzamiento litoral pleistocénico del norte de Chile. Eda- 
des $21 \mathrm{NE}$ de la terraza costera más alta del área de Caldera-Bahía Inglesa. Revista Geológica de Chile, 2007, vol. 34, N 1, p. 81-96.

SHORT, A. D. Waves-dominated beachs. In: SHORT, A. (ed.). Handbook of beach and shoreface morphodynamics. Chichester: John Wiley \& Sons, 1999, p. 173-191.

SOTO, M. V. Análisis de los cambios asociados al oleaje en una ensenada de la zona central de Chile. Anales de la Sociedad Chilena de Ciencias Geográficas, 2003, p. 135-144.
SOTO, M. V. Relaciones morfodinámicas y estructurales en ensenadas desalineadas del litoral de Chile central: Pichilemu y caleta Los Piures. Revista de Geografía Norte Grande, 2005, No33, p. 73-78.

SOTO, M. V. \& ARRIAGADA, J. Características dinámicas de ensenadas estructurales en Chile central. Maitencillo y Papudo, Región de Valparaíso. Revista de Geografía Norte Grande, 2007, №38, p. 99-112. 